\title{
Análisis de insumo-producto de energía y observaciones sobre el desarrollo sustentable, caso mexicano 1970-2010
}

\section{Energy Input-Output Analysis and Remarks on Sustainability Development, Mexican Case 1970-2010}

\author{
Livas-García Adrián \\ Posgrado en Ingeniería (Energía) \\ Universidad Nacional Autónoma de México \\ Correo:adrianlivasgarcia@gmail.com
}

Información del artículo: recibido: noviembre de 2013, aceptado: enero de 2014

\section{Resumen}

La energía para México es un elemento importante de su desarrollo, su adecuada gestión puede contribuir a alcanzar criterios de sustentabilidad. La transformación de energía disponible a energía útil ha aumentado por la producción de hidrocarburos y no por mejoras tecnológicas en la transformación y distribución de energía ${ }^{1}$. Se utiliza el concepto de requerimientos energéticos de energía con la metodología de insumo-producto y el análisis de cambio estructural. Los resultados confirman que la estructura del sistema energético mexicano se ha anclado a los hidrocarburos, y son las exportaciones de energía, los sectores económicos, el residencial y el transporte los principales consumidores. La relación del consumo directo e indirecto con respecto al consumo total ha cambiado de $70 \%-30 \%$ a $40 \%-60 \%$, respectivamente. $80 \%$ del consumo directo está concentrado en los sectores transporte, economía y exportaciones de energía, 97\% del consumo indirecto son las exportaciones de energía, la economía y los medios de transporte, y del consumo total, $83 \%$ se encuentra en las exportaciones de energía, la economía y los medios de transporte. Los resultados del análisis muestran que el efecto de mayor peso para el sistema fueron las variaciones en el uso de energía, y los de menor peso fueron las eficiencias en la transformación de energía y las capacidades de sustitución energética. Además al aplicar el análisis a cada uno de los sectores se observa que las causas de la evolución de su consumo de energía han tenido distintas trayectorias. Se concluye que la principal causa de los cambios directos e indirectos en el consumo de energía lo provocó la producción de hidrocarburos en la década de los 70, aumentando la entropía del sistema energético mexicano. Se identifica la diversificación energética como la opción que tienen las implicaciones positivas de mayor peso para el sistema, al alterar los componentes directos e indirectos del consumo de energía.

1 Se entiende por energía disponible la cantidad de energía producida, después de los procesos de conversión de energía. Y por energía útil aquella que se utilizó en los centros de consumo.

\section{Descriptores:}

- insumo-producto de energía

- requerimientos de energía

- análisis estructural

- consumo de energía

- desarrollo sustentable 


\begin{abstract}
Energy for Mexico is an important element for its development: its proper management can contribute to achieve sustainability criteria. The processing for available energy to useful energy has increased due to the fossil fuel production and not technological improvements in conversion and distribution of energy. The concept of energy requirements of energy is used with the input-output methodology and the structural change analysis. The results confirm that the Mexican energy system structure is coupled to fossil fuel, where energy exports, economics, residential and transportation have been the main consumers. The relationship of the direct and indirect energy consumption in regard to the total has changed from $70 \%-30 \%$ to $40 \%-60 \%$ respectively. The $80 \%$ of direct consumption is concentrated in transportation, economics and energy exports, $97 \%$ of indirect consumption are energy exports, economics and transportation, and in the total consumption, $83 \%$ is concentrated in energy exports, economics and transportation. The analysis results show that the most representative effect was the variations in energy consumption, and the least representative was the efficiency in energy conversion and the energy replacement capabilities. In addition to applying the analysis to each sector it was observed that they have had different trajectories in the causes of their energy consumption evolution. It is concluded that the main cause of direct and indirect changes in energy consumption was the production of hydrocarbons in the decade of the 70's, increasing the entropy of the Mexican energy system. Energy diversification is identified as the option that has the most significant positive implications for the system by altering the direct and indirect components of the total energy consumption.
\end{abstract}

\section{Introducción}

La energía es un bien que tiene un carácter estratégico para todos los Estados nación del mundo. En las últimas décadas se han intensificado todas las actividades relacionadas con la cadena energética y sus consecuencias ambientales de producción y consumo, esto se debe principalmente a la tendencia del agotamiento de los hidrocarburos de fácil acceso, y a las evidencias del cambio climático.

En 1987, la Comisión Mundial sobre Desarrollo y Medio Ambiente publicó el reporte: “Our Common Future", en donde se establece que el desarrollo sustentable es aquel que satisface las necesidades del presente sin comprometer las habilidades de las generaciones futuras para satisfacer las propias (UNDESA, 1987).

La definición se toma en la Cumbre de la Tierra para abordar la necesidad de encontrar puntos de equilibrio entre las necesidades económicas, sociales y ambientales de las generaciones presentes y futuras, (Sheinbaum et al., 2009).

Uno de los aspectos en el debate para alcanzar los criterios del desarrollo sustentable es la energía, ya que su accesibilidad, calidad, nivel tecnológico y estado de la infraestructura, tiene efectos directos en la calidad de vida y en los procesos productivos. Sin embargo, es im- posible dejar de lado que su producción y consumo generan impactos ambientales relevantes a nivel local y global, atentando sobre las capacidades de las generaciones presentes y futuras de todas las especies del planeta, por lo tanto, la energía es un medio que contribuye al desarrollo sustentable (Johanson y Goldemberg, 2002).

Los vínculos entre las tres dimensiones y el consumo de energía se definen mediante las relaciones de: la actividad, que para los sectores económicos es el PIB y para el sector residencial la cantidad de equipamiento en los hogares; la oferta de energía, que es la producción de energía para satisfacer la mezcla energética y el costo energético asociado; el estado de la infraestructura, que se refiere al nivel de accesibilidad más las pérdidas por transmisión, distribución y almacenamiento de energía; y la capacidad de sustitución energética, que se refiere a la capacidad que tienen los centros de consumo final para utilizar diferentes energéticos.

Todas estas se relacionan directamente con aspectos ambientales, cualquier consumo de energía tiene asociado emisiones de gases de efecto invernadero (GEI). Sin embargo, la estructura de las emisiones puede cambiar en función del nivel de consumo, de la cantidad de carbono contenido en la oferta energética, de la eficiencia en los procesos de conversión de energía, de la eficiencia de suministro, etcétera (Flores et al., 2010). 
La propuesta metodológica es construir tablas de insumo-producto de energía, estas tienen la utilidad de representar, bajo el principio de conservación de energía, la trayectoria de cada forma de energía que compone el sistema energético de una región determinada, en un periodo de tiempo establecido.

La metodología se concentra en estimar los requerimientos energéticos de energía (Slesser,1987; Alcántara y Roca, 1995), es decir el costo de la energía para producir energía, y descomponerlos en dos categorías: los relacionados con los procesos de conversión de energía y los asociados a la transmisión-distribución y almacenamiento de energía.

El cálculo se hace en términos de energía primaria para evitar una doble contabilidad de energía y para incluir las características cuantitativas y cualitativas de los centros de consumo, es decir, tomar los efectos globales del sistema (Proops, 1988; Alcántara y Roca, 1995).

La técnica permite determinar las relaciones interenergéticas, que son los nexos de la energía primaria con sus transformaciones en energía secundaria y terciaria.

Una vez construidas las tablas de insumo-producto para diferentes años, la metodología tiene diversas técnicas de análisis, una de ellas es el análisis estructural, en este caso se utiliza para cuantificar los cambios en el consumo de energía a través de la eficiencia de transformación, la capacidad de sustitución y en los niveles de consumo.

La metodología se aplica al caso mexicano con información de los balances de energía para 40 años. La relevancia de este estudio radica en las características propias de México, es decir, como un país que cuenta con vastos recursos energéticos, fósiles y renovables; la producción y utilización de estos ha transitado por diversas etapas en las que han ocurrido cambios en la modalidad de coordinación, en su régimen fiscal, en los métodos de exploración-extracción de petróleo crudo, en las tecnologías de producción de electricidad, en la mezcla energética, en la infraestructura de suministro y sobre todo en los requerimientos energéticos del propio sistema.

Es innegable que la energía para México es un elemento que ha participado con el desarrollo nacional y a la convergencia ideológica de la sociedad, considerándolo un bien nacional, que contribuye con la identidad nacional.
Por lo tanto, la función que cumple el sector energético para México es un elemento clave para encontrar rutas y posibles puntos de equilibrio dirigidos a alcanzar criterios de desarrollo sustentable; la propuesta de este estudio es hacerlo a través de las causas que explican el consumo de energía.

El objetivo principal de este estudio es explicar los cambios en el consumo de energía en México a través del análisis estructural del insumo-producto de energía. Para el análisis se tomó como año base 1970, dado que el interés es evaluar los cambios durante 40 años.

\section{Desarrollo}

Metodología para la construcción del insumo-producto de energía

El insumo-producto de energía se construye según el principio de conservación de energía, está compuesto por tres secciones:

1) Producción de energía o energía total producida.

2) Tansformaciones de energía o energía de demanda intermedia.

3) Centros de consumo final o energía de demanda final, (Alcántara y Roca, 1995; Miller y Blair, 2009; Pruitichaiwiboon et al., 2011),(tabla 1).

La sección de producción de energía está compuesta por la suma de los factores de producción de cada forma de energía, que son: producción nacional, producción que proviene de otras fuentes, importación, variación de inventarios, energía no aprovechada y maquila e intercambio neto.

La sección de energía intermedia está compuesta por los consumos del energético $i$ que se utilizan para la producción del energético $j$, donde la diagonal describe los autoconsumos de cada forma de energía. Por ejemplo, en la intersección del renglón de petróleo crudo
Tabla 1. Estructura de la matriz de insumo-producto de energía

\begin{tabular}{|c|c|c|c|c|c|c|c|c|c|c|c|}
\hline & \multicolumn{5}{|c|}{ Energía Intermedia } & \multicolumn{5}{|c|}{ Energía final } & \multirow{2}{*}{$\begin{array}{c}\text { Energía Total } \\
\text { consumida }\end{array}$} \\
\hline & $\mathrm{e}_{1,1}$ & $\mathrm{e}_{1,2}$ & $\mathrm{e}_{1,3}$ & $\cdots$ & $\mathrm{e}_{1, \mathrm{j}}$ & $\mathrm{e}_{1,1}$ & $\mathrm{e}_{1,2}$ & $\mathrm{e}_{1,3}$ & $\ldots$ & $\mathrm{e}_{1, \mathrm{k}}$ & \\
\hline & $\mathrm{e}_{2,1}$ & $\mathrm{e}_{2,2}$ & $\mathrm{e}_{2,3}$ & $\ldots$ & $\mathrm{e}_{2, \mathrm{j}}$ & $\mathrm{e}_{2,1}$ & $e_{2,2}$ & $e_{2,3}$ & $\ldots$ & $\mathrm{e}_{2, \mathrm{k}}$ & $\mathrm{E}_{2}$ \\
\hline & $\mathrm{e}_{3,1}$ & $\mathrm{e}_{3,2}$ & $\mathrm{e}_{3,3}$ & $\cdots$ & $e_{3, j}$ & $\mathrm{e}_{3,1}$ & $\mathrm{e}_{3,2}$ & $\mathrm{e}_{3,3}$ & $\cdots$ & $\mathrm{e}_{3, \mathrm{k}}$ & $E_{3}$ \\
\hline & $\vdots$ & $\vdots$ & $\vdots$ & $\vdots$ & $\vdots$ & $\vdots$ & $\vdots$ & $\vdots$ & $\vdots$ & $\vdots$ & $\vdots$ \\
\hline & $\mathrm{e}_{\mathrm{i}, 1}$ & $\mathrm{e}_{\mathrm{i}, 2}$ & $e_{i, 3}$ & $\cdots$ & $e_{i, j}$ & $e_{i, 1}$ & $\mathrm{e}_{\mathrm{i}, 2}$ & $e_{i, 3}$ & $\cdots$ & $e_{i, k}$ & $\mathrm{E}_{\mathrm{i}}$ \\
\hline $\begin{array}{l}\text { Factores de } \\
\text { producción }\end{array}$ & $\mathrm{e}_{1}$ & $\mathrm{e}_{2}$ & $\mathrm{e}_{3}$ & $\cdots$ & $e_{j}$ & & & & & & \\
\hline $\begin{array}{l}\text { Energía total } \\
\text { Producida }\end{array}$ & $\mathrm{E}_{1}$ & $\mathrm{E}_{2}$ & $\mathrm{E}_{3}$ & $\cdots$ & $E_{j}$ & & & & & & $E_{i}=E_{j}$ \\
\hline
\end{tabular}


con su columna se tendrá la cantidad de energía consumida para su propia producción y, en ese mismo renglón, en sus intersecciones con las columnas correspondientes a sus derivados (coque de petróleo, gas licuado de petróleo, gasolinas, los productos no energéticos y gas seco) se tendrá la aportación energética para la producción de cada uno de ellos².

La sección de energía final se compone del consumo del energético $i$ en el centro de consumo $k$.

Una vez construidas las tablas de insumo-producto de energía se realiza el análisis básico, este consiste en estimar los requerimientos energéticos de energía directos, indirectos y totales.

Para su construcción se expresan todas las formas de energía como la suma de las cantidades de energía consumida en demanda intermedia más demanda fi$\mathrm{nal}^{3}$, esta tiene que ser igual a la energía total consumida y producida:

$\bar{E}_{\text {intermedia }}+\bar{E}_{\text {final }}=\bar{E}_{\text {total }}$

se estima la proporción de la demanda intermedia con respecto al consumo total

$$
\begin{aligned}
& \overline{\bar{G}} \widehat{E}_{\text {total }}=\bar{E}_{\text {intermedia }} \\
& \overline{\bar{G}}=\bar{E}_{\text {intermedia }} \widehat{E}_{\text {total }}^{-1}
\end{aligned}
$$

el cociente (3) es el enlace para explicar un cambio en la energía total como una causalidad de la estructura interna del sistema energético, se conoce como la matriz de requerimientos directos de energía (RDE) ${ }^{4}$. Se sustituye (2) en (1):

$\overline{\bar{G}} \widehat{E}_{\text {total }}+\bar{E}_{\text {final }}=\bar{E}_{\text {total }} \Rightarrow \bar{E}_{\text {total }}=(\overline{\bar{I}}-\overline{\bar{G}})^{-1} \bar{E}_{\text {final }}$

La matriz $(\overline{\bar{I}}-\overline{\bar{G}})^{-1}$ para este trabajo es la Inversa de Leontief de Energía, $\overline{I L E}$, y expresa los requerimientos totales de energía (RTE) ${ }^{5}$.

La diferencia entre los RTE y los RDE son los requerimientos indirectos de energía (RIE):

$$
[\overline{\overline{I L E}}-\overline{\overline{\mathrm{G}}}]
$$

2 Los autoconsumos se conforman del autoconsumo, pérdidas por transmisión, distribución y almacenamiento.

$3\left(\overline{\mathrm{E}}_{\text {intemmedia }}\right)=\sum_{i}^{n} e_{i, j}$ es el resultado de la suma de cada tipo de energía en la matriz de transformaciones de energía $\left(\overline{\bar{E}}_{\text {intremedia }}\right)$. y $\left(\overline{\bar{E}}_{\text {intremedia }}\right) \cdot Y, \bar{E}_{\text {frual }}=\sum_{i}^{n} e_{i, k}$ es el resultado de la suma de cada tipo de energía en los centros de consumo de energía final $\left(\overline{\mathrm{E}}_{\text {final }}\right)$.

4 Los RDE, son las proporciones de las diferentes formas de energía consumida directamente en los procesos de conversión de energía, por lo que expresa la tabla de transformaciones, también se les conoce como los coeficientes de transformación de energía.

5 Los RTE son la proporción de energía que debe producirse para abastecer los centros de consumo, considerando todo el conjunto pérdidas.
Hasta este punto la $\bar{E}_{\text {total }}$ tiene doble contabilidad, esto se debe a la conversión de energía primaria a secundaria y terciaria, la proporciones de transformación que se encuentran en los RTE, RDE, y RIE. Para ajustarla, se toman solamente los renglones correspondientes a energía primaria, (Alcántara y Roca, 1995).

De este modo la energía total se obtiene en términos de energía primaria, incluyendo las características cuantitativas y cualitativas de todos los centros de consumo más los efectos de los mercados internacionales de energía primaria (Proops, 1988).

El consumo de energía primaria total se descompone en directo e indirecto:

$$
\begin{aligned}
& \overline{E P}_{\text {total }}=\overline{\overline{I L E}}_{p} \bar{E}_{\text {final }} \\
& \overline{E P}_{\text {directa }}=\overline{\bar{G}}_{p} \bar{E}_{\text {final }} \\
& \overline{E P}_{\text {indirecta }}=(\overline{\overline{I L E}}-\overline{\bar{G}})_{p} \bar{E}_{\text {final }}
\end{aligned}
$$

\section{Metodología del análisis de cambio estructural}

El análisis de cambio estructural (ACE) mide los cambios como la suma de cuatro efectos:

1. Eficiencia de transformación de energía (ETE), son las pérdidas en los procesos de producción, distribución y almacenamiento de energía ${ }^{6}$.

2. Las formas de sustitución de las diferentes opciones de energía en el consumo final (SE) ${ }^{7}$.

3. Variaciones en el uso de la energía en los centros de consumo (VUE). Esas variaciones son resultado de diferentes factores como en el caso de la demanda de energía final de los sectores económicos, son el efecto de la actividad (PIB de cada rama) y la estructura energética; en el caso de la demanda residencial, los efectos dependen de la cantidad de equipamiento de los hogares y la eficiencia de obtención de energía; para el sector transporte la demanda depende del nivel de actividad y de la eficiencia energética en términos de transporte ${ }^{8}$.

4. El último efecto es de interacción, que se interpreta como un residuo no explicable (EI).

\footnotetext{
6 La evidencia de mejora del sistema energético por la eficiencia de transformación de energía y menores pérdidas globales se tiene con valores negativos.

7 Valores positivos implican que al aumento en la demanda de energía se ha cubierto con aumento de la diversificación energética.

8 El porcentaje puede ser mayor que $100 \%$ porque se están contabilizadas las pérdidas (eficiencias de transformación ni en transporte, distribución y almacenamiento).
} 
Las expresiones para cuantificar cada efecto son

$\Delta \overline{\overline{E P}}_{\text {total }}=\overline{\overline{E P}}_{\text {total }, t}-\overline{\overline{E P}}_{\text {total }, 0}=\overline{\overline{E T E}}_{t}+\overline{\overline{S E}}_{t}+\overline{\overline{V U E}}_{t}+\overline{\overline{E I}}_{t}$

donde

$$
\begin{aligned}
& \overline{\overline{E T E}}_{t}=\left(\overline{\overline{I L E}}_{t}-\overline{\overline{I L E}}_{0}\right) \overline{\overline{\mathrm{A}}}_{0} \widehat{\mathrm{B}}_{0} \\
& \overline{\overline{S E}}_{t}=\overline{\overline{I L E}}_{0}\left(\overline{\bar{A}}_{t}-\overline{\bar{A}}_{0}\right) \widehat{\mathrm{B}}_{0} \\
& \overline{\overline{\operatorname{VUE}}_{t}}=\overline{\overline{\overline{I L E}}_{0}} \overline{\bar{A}}_{0}\left(\widehat{\mathrm{B}}_{t}-\overline{\bar{B}}_{0}\right)
\end{aligned}
$$

Las matrices $\overline{\bar{A}}$ y $\widehat{B}$, provienen de la descomposición de la matriz $\overline{\bar{E}}_{\text {final }}=\overline{\bar{A}} \widehat{B}$, la primera es la proporción de las diferentes formas de energía con respecto a la demanda final de cada centro de consumo y la segunda es un vector diagonal que expresa la demanda final de energía de cada centro de consumo9.

\section{Caso mexicano}

Para desarrollar la metodología se utiliza la información publicada por la Secretaria de Energía en su área de Sistema de Información Energética, que es la autoridad que presta el servicio público de información estadística en materia energética (SENER).

La nomenclatura utilizada es: C, carbón; PC, petróleo crudo; GN, gas natural; $N$, nuclear; $H$, hidroenergía; ER, energías renovables; $\mathrm{B}$, biomasa; $\mathrm{CC}$, coque de carbón; $\mathrm{CP}$, coque de petróleo; GLP, gas licuado de petróleo; G, gasolinas; PNE, productos no energéticos; GS, gas seco; E, electricidad; Ex, exportaciones; CNE, consumo no energético; $\mathrm{R}$, residencial; $\mathrm{T}$, transporte; A, agropecuario; Ec, económicos; Ept, energía producida en centros de transformación; $\mathrm{P}$, producción de energía; POF, producción de energía de otras fuentes; Im, importación; VI, variación de inventarios; NA, energía no aprovechada; MIN, maquila e intercambio neto; EI, energía intermedia; EF, energía final; y ET, energía total ${ }^{10}$.

La metodología inicia con la construcción de las tablas de insumo-producto de energía. El consumo y producción de energía por tipo de energético para el año 2010 se presenta en la tabla 2 (note el principio de con-

9 La matriz $\overline{\bar{E}}_{\text {final }}$ proviene de los elementos $e_{i, k}$.

10 Para facilitar la lectura se agregaron las cuentas por tipos de energía: PC, petróleo crudo y condensados; ER, geoenergía, solar y eólica; B, bagazo de caña y leña; y G, gasolina y naftas, querosenos, diesel, y combustóleo. Las cuentas de los centros de consumo se agregaron para: $\mathrm{CNE}$, petroquímica de PEMEX y otras ramas económicas; $\mathrm{T}$, aéreo, autotransporte, eléctrico, ferroviario, y marítimo; y EC comercio, público, petroquímica PEMEX, aguas envasadas, aluminio, automotriz, azúcar, celulosa y papel, cemento, cerveza y malta, construcción, fertilizantes, hule, minería, química, siderurgia, tabaco, vidrio, y otras ramas económicas. servación de energía). La cantidad de energía producida es igual a la consumida, también se tienen las cantidades de energía consumida para la producción de energía y en la diagonal de la tabla de transformaciones ( $\bar{E}_{\text {intermedia }}$ ) están los autoconsumos de cada energético.

El siguiente paso es calcular los $\mathrm{RDE}^{11}$, la tabla 3 muestra la matriz $\overline{\bar{G}}_{2010}$, las lecturas son: 1) del lado del consumo, están las unidades de energía del energético $i$ utilizadas para la producción de sus subproductos $j s ; 2$ ) por el lado de la producción, se tienen las unidades de energía de los energéticos is necesarias para la producción del energético $j$.

Después se estiman los RTE, la tabla 4 (matriz $\overline{\overline{I L E}}_{2010}$ brinda información de las proporciones totales de energía para producir energía, considerando la consumida directamente en los procesos de conversión más todo el conjunto de pérdidas asociadas con colocar cada energético en los centros de consumo.

Para ajustar la doble contabilidad se toman solamente los renglones correspondientes a energía primaria, los primeros siete de la tabla 4 .

De ahí se calcula la matriz $\overline{E P}_{\text {total }, 2010}$, la cantidad de energía necesaria para abastecer la demanda de energía o las cantidades de energía consumida en los diferentes centros de consumo en términos de energía primaria. La matriz aproxima la cantidad total de energía necesaria tomando en cuenta las eficiencias de transformación y todo el conjunto de pérdidas (tabla 5).

La tabla 6 muestra los resultados de la evaluación del ACE para el año 2010.

\section{Discusión y análisis de resultados}

La metodología se desarrolla en dos etapas, la primera para el caso del año 2010, un caso estático, y la segunda para el periodo de tiempo de 1970 al 2010, un caso dinámico.

\section{Caso estático}

El insumo-producto de energía para el año 2010 (tabla $2)$, muestra las relaciones interenergéticas de todos los tipos de energía. El petróleo crudo consume 31.3 PJ para su propia producción, más 48.4, 59.3, 2496, 103 y 160.4 PJ para la producción de coque de petróleo, gas licuado de petróleo, gasolinas, productos no energéticos y gas seco, respectivamente. También se observa el rendimiento energético, en este caso se tiene que por cada unidad de energía consumida para su producción se obtienen 195 unidades de energía ${ }^{12}$.

\footnotetext{
11 También conocidos como coeficientes de transformación. 12 Rendimiento energético es el cociente de la energía necesaria para su propia producción y el total de la energía producida.
} 
Tabla 2. Insumo-producto de energía 2010 (PJ)

\begin{tabular}{|c|c|c|c|c|c|c|c|c|c|c|c|c|c|c|c|c|c|}
\hline & C & PC & GN & $\mathrm{N}$ & $\mathrm{H}$ & ER & B & $\mathrm{CC}$ & $\mathrm{CP}$ & GLP & G & PNE & GS & $\mathrm{E}$ & EI & $\mathrm{EF}$ & ET \\
\hline C & - & - & - & - & - & - & - & 63.3 & - & - & - & - & - & 322.7 & 386.1 & 13.0 & 399.1 \\
\hline PC & - & 31.3 & - & - & - & - & - & - & 48.4 & 59.3 & $2,496.0$ & 103.0 & 160.4 & - & $2,898.4$ & $3,198.4$ & $6,096.8$ \\
\hline GN & - & - & 230.3 & - & - & - & - & - & - & 297.3 & 150.5 & 89.0 & $1,331.1$ & - & $2,098.2$ & 710.4 & $2,808.5$ \\
\hline $\mathrm{N}$ & - & - & - & - & - & - & - & - & - & - & - & - & - & 63.9 & 63.9 & - & 63.9 \\
\hline $\mathrm{H}$ & - & - & - & - & - & - & - & - & - & - & - & - & - & 132.3 & 132.3 & - & 132.3 \\
\hline ER & - & - & - & - & - & - & - & - & - & - & - & - & - & 150.5 & 150.5 & 4.9 & 155.4 \\
\hline B & - & - & - & - & - & - & - & - & - & - & - & - & - & - & - & 347.3 & 347.3 \\
\hline CC & - & - & - & - & - & - & - & 3.5 & - & - & - & - & - & - & 3.5 & 63.3 & 66.8 \\
\hline $\mathrm{CP}$ & - & - & - & - & - & - & - & - & - & - & - & - & - & - & - & 116.8 & 116.8 \\
\hline GLP & - & - & - & - & - & - & - & - & - & 4.9 & - & - & - & - & 4.9 & 450.2 & 455.1 \\
\hline G & - & - & - & - & - & - & - & - & - & - & 115.1 & - & - & 391.9 & 507.0 & $2,919.2$ & $3,426.1$ \\
\hline PNE & - & - & - & - & - & - & - & - & - & - & - & - & - & - & - & 175.9 & 175.9 \\
\hline GS & - & - & - & - & - & - & - & - & - & - & - & - & 452.0 & 985.1 & $1,437.0$ & 479.6 & $1,916.6$ \\
\hline E & - & - & - & - & - & - & - & - & - & - & - & - & - & 199.2 & 199.2 & 675.3 & 874.6 \\
\hline Ept & - & 31.3 & 230.3 & - & - & - & - & 66.9 & 48.4 & 361.5 & $2,761.6$ & 191.9 & $1,943.5$ & $2,245.6$ & & & \\
\hline $\mathrm{P}$ & 202.6 & $6,101.1$ & $2,247.0$ & 63.9 & 132.3 & 155.4 & 348.3 & 58.6 & 43.7 & 330.6 & $2,395.4$ & 175.9 & $1,385.2$ & 873.1 & & & \\
\hline POF & - & - & 733.3 & - & - & - & - & - & - & - & - & - & - & - & & & \\
\hline Im & 201.2 & - & - & - & - & - & - & 10.4 & 83.2 & 122.4 & $1,046.8$ & - & 521.9 & 1.4 & & & \\
\hline VI & -4.7 & -4.0 & -2.7 & - & - & - & - & -2.2 & -10.1 & 2.1 & -16.0 & -0.0 & 9.5 & - & & & \\
\hline NA & - & 0.4 & -169.0 & - & - & - & -1.0 & - & - & - & - & - & - & - & & & \\
\hline MIN & - & - & - & - & - & - & - & - & - & - & - & - & - & - & & & \\
\hline ET & 399.1 & $6,097.5$ & $2,808.5$ & 63.9 & 132.3 & 155.4 & 347.3 & 66.8 & 116.8 & 455.1 & $3,426.1$ & 175.9 & $1,916.6$ & 874.6 & & & \\
\hline
\end{tabular}

Tabla 3. Matriz $\overline{\bar{G}}_{2010}$, requerimientos directos de energía

\begin{tabular}{|c|c|c|c|c|c|c|c|c|c|c|c|c|c|c|}
\hline & C & PC & GN & $\mathrm{N}$ & $\mathrm{H}$ & ER & B & CC & $\mathrm{CP}$ & GLP & G & PNE & GS & E \\
\hline C & - & - & - & - & - & - & - & 0.9 & - & - & - & - & - & 0.4 \\
\hline PC & - & 0.0 & - & - & - & - & - & - & 0.4 & 0.1 & 0.7 & 0.6 & 0.1 & - \\
\hline GN & - & - & 0.1 & - & - & - & - & - & - & 0.7 & 0.0 & 0.5 & 0.7 & - \\
\hline $\mathrm{N}$ & - & - & - & - & - & - & - & - & - & - & - & - & - & 0.1 \\
\hline $\mathrm{H}$ & - & - & - & - & - & - & - & - & - & - & - & - & - & 0.2 \\
\hline ER & - & - & - & - & - & - & - & - & - & - & - & - & - & 0.2 \\
\hline B & - & - & - & - & - & - & - & - & - & - & - & - & - & - \\
\hline CC & - & - & - & - & - & - & - & 0.1 & - & - & - & - & - & - \\
\hline $\mathrm{CP}$ & - & - & - & - & - & - & - & - & - & - & - & - & - & - \\
\hline GLP & - & - & - & - & - & - & - & - & - & 0.0 & - & - & - & - \\
\hline G & - & - & - & - & - & - & - & - & - & - & 0.0 & - & - & 0.4 \\
\hline PNE & - & - & - & - & - & - & - & - & - & - & - & - & - & - \\
\hline GS & - & - & - & - & - & - & - & - & - & - & - & - & 0.2 & 1.1 \\
\hline E & - & - & - & - & - & - & - & - & - & - & - & - & - & 0.2 \\
\hline
\end{tabular}


Tabla 4. Requerimientos totales de energía

\begin{tabular}{|c|c|c|c|c|c|c|c|c|c|c|c|c|c|c|}
\hline & C & PC & GN & $\mathrm{N}$ & $\mathrm{H}$ & ER & B & $\mathrm{CC}$ & $\mathrm{CP}$ & GLP & $G$ & PNE & GS & $E$ \\
\hline $\mathrm{C}$ & 1.0 & - & - & - & - & - & - & 1.0 & - & - & - & - & - & 0.5 \\
\hline PC & - & 1.0 & - & - & - & - & - & - & 0.4 & 0.1 & 0.8 & 0.6 & 0.1 & 0.6 \\
\hline GN & - & - & 1.1 & - & - & - & - & - & - & 0.7 & 0.0 & 0.6 & 1.0 & 1.5 \\
\hline $\mathrm{N}$ & - & - & - & 1.0 & - & - & - & - & - & - & - & - & - & 0.1 \\
\hline $\mathrm{H}$ & - & - & - & - & 1.0 & - & - & - & - & - & - & - & - & 0.2 \\
\hline ER & - & - & - & - & - & 1.0 & - & - & - & - & - & - & - & 0.2 \\
\hline B & - & - & - & - & - & - & 1.0 & - & - & - & - & - & - & - \\
\hline CC & - & - & - & - & - & - & - & 1.1 & - & - & - & - & - & - \\
\hline $\mathrm{CP}$ & - & - & - & - & - & - & - & - & 1.0 & - & - & - & - & - \\
\hline GLP & - & - & - & - & - & - & - & - & - & 1.0 & - & - & - & - \\
\hline G & - & - & - & - & - & - & - & - & - & - & 1.0 & - & - & 0.6 \\
\hline PNE & - & - & - & - & - & - & - & - & - & - & - & 1.0 & - & - \\
\hline GS & - & - & - & - & - & - & - & - & - & - & - & - & 1.3 & 1.9 \\
\hline $\mathrm{E}$ & - & - & - & - & - & - & - & - & - & - & - & - & - & 1.3 \\
\hline
\end{tabular}

Tabla 5. Matriz $\overline{\overline{E P}}_{\text {total }, 2010}(\mathrm{PJ})$

\begin{tabular}{lllllllll}
\hline & & & & & & & \multicolumn{2}{c}{$\%$ del } \\
Ex & CNE & \multicolumn{1}{c}{$\mathrm{R}$} & \multicolumn{1}{c}{$\mathrm{T}$} & \multicolumn{1}{c}{$\mathrm{A}$} & \multicolumn{1}{c}{ Ec } & EP & total \\
\hline $\mathrm{C}$ & 2.5 & - & 85.0 & 2.1 & 14.8 & 290.7 & 395.1 & 0.04 \\
$\mathrm{PC}$ & $3,589.3$ & 140.2 & 188.9 & $1,423.7$ & 118.9 & 628.5 & $6,089.5$ & 0.65 \\
$\mathrm{GN}$ & 50.3 & 117.5 & 496.6 & 180.6 & 49.2 & $1,293.5$ & $2,187.7$ & 0.23 \\
$\mathrm{~N}$ & 0.5 & - & 16.8 & 0.4 & 2.9 & 43.8 & 64.5 & 0.01 \\
$\mathrm{H}$ & 1.0 & - & 34.8 & 0.9 & 6.1 & 90.7 & 133.4 & 0.01 \\
$\mathrm{ER}$ & 1.1 & - & 42.4 & 1.0 & 6.9 & 105.3 & 156.7 & 0.02 \\
$\mathrm{~B}$ & - & 0.2 & 259.3 & - & - & 87.8 & 347.3 & 0.04 \\
\hline Total & $3,644.5$ & 257.9 & $1,123.9$ & $1,608.6$ & 198.8 & $2,540.4$ & $9,374.2$ & \\
\hline \% del total & 0.39 & 0.03 & 0.12 & 0.17 & 0.02 & 0.27 & & \\
\hline
\end{tabular}

Nota. $E P_{i}$ es el consumo de energía primaria de tipo $i, y E P_{k}$ es el consumo de energía en del centro de consumo $k$

Tabla 6. Efectos que explican los cambios en el consumo de energía primaria (PJ)

\begin{tabular}{llllllc}
\hline & $\Delta \mathrm{EP}$ & ETE & SE & VUE & EI & Promedio \\
\hline Ex & $3,428.5$ & -11.4 & -32.1 & $4,123.6$ & -651.6 & $1,371.4$ \\
CNE & 164.8 & -13.8 & -2.8 & 228.4 & -47.0 & 65.9 \\
R & 797.1 & 0.9 & 120.9 & 488.0 & 187.3 & 318.8 \\
T & $1,149.2$ & -164.8 & 0.1 & $2,058.5$ & -744.5 & 459.7 \\
A & 132.4 & -8.4 & 12.8 & 130.3 & -2.3 & 52.9 \\
Ec & $1,782.0$ & -74.6 & 128.1 & $1,568.1$ & 160.3 & 712.8 \\
\hline Total & $7,454.0$ & -272.2 & 227.0 & $8,596.9$ & $-1,097.7$ & $2,981.6$ \\
\hline
\end{tabular}


Después se construyeron los RDE (tabla 3), las proporciones de energía necesarias para los procesos de conversión de energía. Para transformar carbón en los subproductos es necesario que se suministren al sistema de transformación 1.3 unidades de energía, dado que 0.9 se transformaron en coque de carbón y 0.4 en electricidad; otra forma, la eficiencia de transformación de carbón en coque de carbón es de $90 \%$ y de $40 \%$ para convertirlo en electricidad.

En la tabla 4 están los RTE, las proporciones de energía necesarias para llevar a cabo los procesos de conversión de energía para colocar una unidad de energía como producto final en los centros de consumo.

De la matriz de RDT, los elementos que componen el vector de electricidad son interesantes por tener la mezcla energética más amplia, por ejemplo, para producir una unidad de energía eléctrica se requieren 0.5, $0.6,1.5,0.2,0.2,0.6,1.9$ y 1.3 unidades de energía de carbón, petróleo crudo, gas natural, nuclear, hidroenergía, energías renovables, gas licuado de petróleo, gas seco, y electricidad, respectivamente. De otra forma, para producir una unidad de energía eléctrica se requieren de 6.9 unidades de energía de diferentes fuentes, donde 1.3 unidades son propias de la electricidad, además se aprecia la participación de los hidrocarburos y sus productos derivados, que para el gas licuado de petróleo y el gas seco son energía secundaria y la electricidad para producir electricidad se convierte en energía terciaria. En términos de las leyes de la termodinámica, aumentan los pasos de conversión de energía y con ello la entropía del sistema, del mismo modo la ley de la degradación de la energía también lo hace.

Se estimó el consumo de energía en términos de energía primaria (tabla 5). La distribución porcentual de los consumos de energía en los diferentes sectores son: exportación $(39 \%)$, económicos $(27 \%)$, transporte $(17 \%)$, residencial (12\%), CNE (3\%), y agropecuario (2\%).

La mezcla energética para todo el sistema es principalmente petróleo crudo (65\%) y al gas natural (23\%). El petróleo crudo es el principal componente para los sectores exportación $(98 \%)$, CNE $(58 \%)$, transporte $(89 \%)$ y agropecuario $(60 \%)$, y el gas natural para los sectores residencial (44\%) y los económicos (51\%).

En la tabla 6 se encuentran los resultados del ACE para el año 2010, los cambios en el consumo de energía de los diferentes sectores son: exportación (3428.5 $\mathrm{PJ})$, económicos (1782 PJ), transporte (1149.2 PJ), residencial (797.1 PJ), CNE (164.8 PJ) y agropecuario (132.4 PJ).

El primer término del análisis es ETE, además de informar acerca del conjunto de pérdidas de energía también lo hace al identificar las rutas que toma el sector energético para la producción de electricidad y los productos derivados del petróleo, esto en función de las necesidades de energía de los centros de consumo.

Los valores del término son negativos para todos los sectores (excepto para el residencial), esto significa que los coeficientes de transformación del 2010 han aumentado ligeramente con respecto a 1970, en otras palabras, la eficiencia de conversión de energía en el sistema energético mexicano creció, y las pérdidas por transmisión, almacenamiento y distribución disminuyeron.

El sector transporte tiene el valor más negativo, es decir, los coeficientes de transformación mayores y las pérdidas asociadas menores. Caso contrario para el sector residencial, único con un valor positivo (cercano a la unidad). Esto quiere decir que el conjunto de pérdidas aumentó con respecto a 1970.

El siguiente término es $\mathrm{SE}$, sustitución de diferentes opciones de energía en los diferentes sectores. Los sectores exportación y CNE tienen valores negativos, esto se entiende, ya que las materias primas para sus actividades no son intercambiables, no es posible diversificar su oferta energética. Sin embargo, para los demás sectores los valores son positivos, esto quiere decir que han diversificado sus opciones energéticas.

Para el sector residencial el componente SE explica $15 \%$ del total de sus cambios en el consumo de energía, mientras que para el sector agropecuario $10 \%$, los sectores económicos $7 \%$ y el transporte $0.01 \%$.

El efecto de mayor peso que explica el cambio en el consumo de energía es VUE, las variaciones en el uso de energía, en otras palabras, la actividad de cada sector aumentó: las exportaciones de energía primaria; los kilómetros recorridos en cada tipo de transporte, al igual que los pasajeros y mercancías transportadas; para los sectores económicos, en esencia su producción; en el sector residencial, además del aumento de hogares, ha crecido la cantidad de aparatos consumidores de energía, y cambios en la energía compartida.

Se observa que los sectores en los que no es posible diversificar sus fuentes energéticas ${ }^{13}$, tienen valores altos en VUE, donde su peso porcentual en el cambio total de energía es mayor a la unidad. Por el contrario, los sectores que presentan evidencias de diversificación energética, tienen valores porcentuales menores a la unidad, en el sector residencial $61 \%$ de los cambios se explican por variaciones en el consumo de energía, $98 \%$ el agropecuario y $88 \%$ los sectores económicos.

El último término es el residual EI, este tiene valores positivos solamente para los sectores residencial y

13 Se refiere a los sectores exportación, CNE, y en menor medida el sector transporte. 
agropecuario, explica el $24 \%$ y $9 \%$ de los cambios en el consumo de energía, respectivamente.

\section{Caso dinámico}

Para el periodo de estudio, 1970 al 2010, se obtuvo el comportamiento de la $\overline{E P}_{\text {total }}$ como la suma de sus componentes directos e indirectos por tipo de energía y por centro de consumo, también se realizó el ACE tomando como año base 1970.

En 40 años la $\overline{E P}_{\text {total }}$ por tipo de energético tuvo un crecimiento porcentual de $388 \%$ (7454 PJ), en la distribución de los consumos de energía el petróleo crudo y el gas natural son los de mayor peso, con una participación porcentual de $68 \%$ (5091 PJ) y 22\% (1636 PJ), respectivamente, (tabla 7) para detallar ${ }^{14}$.

La energía asociada a los procesos de conversión de energía, $\overrightarrow{E P}_{\text {directa }}$, cambió 194\% (2520 PJ), la distribución en el cambio de energía el petróleo crudo y el gas natural tienen las participaciones más grandes, 57\% (1430 PJ) y $24 \%$ (609 PJ).

El cambio en la energía relacionada con el autoconsumo, transmisión, distribución y almacenamiento, $\overline{E P}_{\text {indirecta }}$, fue de $787 \%$ (4934 PJ), en su distribución 74\% (3660 PJ)fueron para el petróleo crudo y 21\% (1027 PJ) para el gas natural.

Tomando en cuenta el balance de energía, los cambios totales en el consumo de energía para los centros de consumo son los mismos. Las distribuciones son: $\overline{E P}_{\text {total }}, 46 \%$ (3429 PJ) es para el sector exportación, 24\% (1782 PJ) para los económicos y 15\% (1149 PJ) para el transporte; $\overline{E P}_{\text {directa }}, 44 \%(1120 \mathrm{PJ})$ de transporte, 25\% (634 PJ) de los económicos, 12\% (302 PJ) del resi-

14 El cambio porcentual de mayor peso es para las energías renovables y la nuclear, sin embargo, en energía no tienen el peso de los hidrocarburos. dencial; $\overline{E P}_{\text {indirecta }}, 64 \%$ (3198 PJ) para exportación, 23\% (1148 PJ) para los económicos, 10\% (494 PJ) del residencial (tabla 8).

La distribución entre el consumo directo e indirecto para el periodo de estudio fue de $34 \%$ y $66 \%$, respectivamente, sin embargo, la evolución porcentual con respecto al total muestra que de 1970 a 1979 el consumo directo era el principal, después el consumo indirecto se colocó como el mayor (figura 1).

$\mathrm{Al}$ aplicar el ACE a todo el periodo de estudio, en el consumo global de energía se observa que los cambios han transitado por cuatro etapas (1970-1982), crecimiento con comportamiento exponencial de $6300 \mathrm{PJ}$; 1982-1995, cambios prácticamente constantes de 6400 PJ; 1995-2007, dos aumentos de 1370 PJ en promedio; y 2007-2010, inicia un descenso de 340 PJ en promedio.

El comportamiento por efecto fue ETE, tiene valores de poco peso con respecto a los demás efectos, cambió de positivo a negativo entre 1990 y 1992, esto quiere decir que aumentó ligeramente la eficiencia de transformación de energía y el conjunto de pérdidas; SE, sustitución de las diferentes opciones de energía, ha tenido valores positivos pequeños en proporción, en las últimas dos décadas tuvo un ligero crecimiento, en otras palabras, el sistema energético está fuertemente anclado a fuentes de energía específicas, los hidrocarburos; VUE es el de mayor peso, las actividades y las características propias de cada sector están fuertemente relacionadas con su consumo de energía; EI, efecto interacción o residual, su valor ha crecido durante todo el periodo (figura 2).

El valor promedio de los cambios en el consumo de energía crece con un comportamiento tipo exponencial en la primera década, las siguientes tienen una trayectoria lineal con una pendiente cercana a cero.

Tabla 7. Cambio en energía y porcentual por tipo de energético; $\overline{E P}_{\text {total }}, \overline{E P}_{\text {directa }}, \overline{E P}_{\text {indirecta }}$ 1970-2010

\begin{tabular}{lllllllllc}
\hline & \multicolumn{3}{c}{ Cambio en energía (PJ) } & \multicolumn{3}{c}{ Cambio porcentual (\%) } \\
\hline C & Total & $\%$ & Directa & $\%$ & Indirecta & $\%$ & Total & Directa & Indirecta \\
PC & 349.9 & 4.7 & 269.0 & 10.7 & 81.0 & 1.6 & 774.9 & 634.2 & $2,947.9$ \\
GN & $5,090.5$ & 68.3 & $1,430.5$ & 56.8 & $3,660.0$ & 74.2 & 509.5 & 171.5 & $2,217.7$ \\
N & $1,635.7$ & 21.9 & 608.5 & 24.1 & $1,027.2$ & 20.8 & 296.3 & 169.7 & 531.0 \\
H & 64.5 & 0.9 & 49.8 & 2.0 & 14.7 & 0.3 & $1,538.5$ & $1,448.4$ & $1,941.1$ \\
ER & 63.3 & 0.8 & 44.9 & 1.8 & 18.4 & 0.4 & 90.3 & 77.3 & 153.4 \\
B & 156.7 & 2.1 & 117.2 & 4.7 & 39.5 & 0.8 & $3,286.5$ & $2,935.6$ & $5,056.1$ \\
\hline Total & 93.3 & 1.3 & - & - & 93.3 & 1.9 & 36.7 & - & 36.7 \\
\hline
\end{tabular}

Nota. Los años de comparación para nucleoenergía, geoenergía, energía solar y energía eólica son respectivamente 1989, 1973, 1990 y 1994 
Para profundizar en el análisis del consumo de energía se desagrega el ACE en los seis sectores considerados, los cambios fueron diferentes para todos, las distribuciones porcentuales en promedio del $\triangle \mathrm{EP}$ son: exportación $48 \%$, económicos $23 \%$, transporte $15 \%$, residencial 9\%, CNE 3\%, y agropecuario 2\%. También las velocidades de crecimiento del cambio en el consumo de energía fueron diferentes para todas las ramas, la mayor fue para el sector residencial, que fue el único sector que duplicó la magnitud y en menor medida los económicos, después transporte, exportación, agropecuario y en trayectoria negativa CNE (tabla 9).

El primer término de la descomposición es ETE, los resultados muestran que existió una ligera mejora en el sistema, de manera que $-1 \%$ del $\triangle E P$ lo explica. Al desagregar el sistema en sus centros de consumo para el sector transporte es de $-6 \%$, CNE $-5 \%$, agropecuario $-2 \%$, exportación $-0.4 \%$, residencial $3.4 \%$ y económicos $0.7 \%$, pero en aumento de pérdidas globales.

El siguiente término SE, durante el periodo de análisis, $2 \%$ de los cambios en el consumo de energía primaria total se explican como resultado de diversificar las fuentes de energía para abastecer la demanda, para los centros de consumo, el sector agropecuario tiene $12 \%$, residencial $11 \%$, económicos $6 \%$, transporte con $0.1 \%$, exportaciones $-1.2 \%$ y CNE $-0.4 \%$, los valores ne- gativos representan las características propias de esos sectores, es decir, no es posible la diversificación.

El VUE es el efecto de mayor peso, durante las cuatro décadas de estudio los cambios en el $\triangle \mathrm{EP}$ del sistema representan $108 \%$, mientras que para los centros de consumo son: transporte $130 \%$, exportaciones $120 \%$, CNE $119 \%$, económicos $83 \%$, agropecuario $80 \%$ y residencial $66 \%$.

El último término, el residual (EI), tiende a aumentar conforme se amplía el tiempo de análisis, para el sistema tiene un peso de $-9 \%$ y para los diferentes sectores son: residencial $20 \%$, agropecuario $10 \%$, económi$\cos 10 \%$, exportaciones $-19 \%$ y transporte $-24 \%$.

La trayectoria de los diferentes efectos es distinta para cada uno de los sectores, aunque el VUE es el de mayor peso. El efecto SE solo se presenta en los sectores residencial, agropecuario y económico; también en estos, el valor del efecto EI es positivo. El efecto ETE es el de menor peso para los tres sectores y en todos se aprecia un cambio de comportamiento en la primera mitad de la década de los 90, cambiando de valores positivos a negativos y nulos; los valores de VUE a pesar de ser el efecto de mayor peso, tiene distintas trayectorias para cada uno de estos sectores; el sector residencial ha crecido constantemente, el agropecuario creció rápidamente hasta 1982 y después tuvo algunas variaciones

Tabla 8. Cambio en energía y porcentual por centro de consumo; $\overline{E P}_{\text {total }}, \overline{E P}_{\text {directa }}, \overline{E P}_{\text {indirecta }}$ 1970-2010

\begin{tabular}{llllllllll}
\hline & \multicolumn{3}{c}{ Cambio en energía (PJ) } & \multicolumn{3}{c}{ Cambio porcentual (\%) } \\
\hline & Total & $\%$ & Directa & $\%$ & Indirecta & $\%$ & Total & Directa & Indirecta \\
\hline Ex & $3,428.5$ & 46.0 & 231.8 & 9.2 & $3,196.7$ & 64.8 & $1,586.8$ & 121.0 & $13,031.8$ \\
CNE & 164.8 & 2.2 & 165.5 & 6.6 & -0.7 & -0.0 & 176.9 & 213.4 & -4.8 \\
R & 797.1 & 10.7 & 302.7 & 12.0 & 494.4 & 10.0 & 243.9 & 348.6 & 206.0 \\
T & $1,149.2$ & 15.4 & $1,119.9$ & 44.4 & 29.4 & 0.6 & 250.2 & 262.1 & 91.5 \\
A & 132.4 & 1.8 & 65.8 & 2.6 & 66.5 & 1.3 & 199.2 & 130.5 & 416.2 \\
Ec & $1,782.0$ & 23.9 & 634.2 & 25.2 & $1,147.8$ & 23.3 & 235.0 & 138.1 & 383.8 \\
\hline Total & $7,454.0$ & 1.0 & $2,519.9$ & 1.0 & $4,934.0$ & 1.0 & 388.2 & 194.9 & 786.6 \\
\hline
\end{tabular}

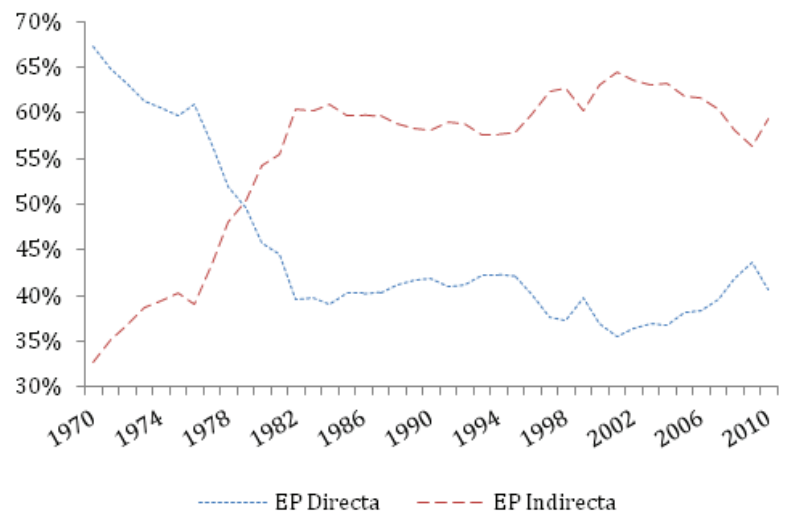

Figura 1. Evolución porcentual del consumo de energía directa e indirecta

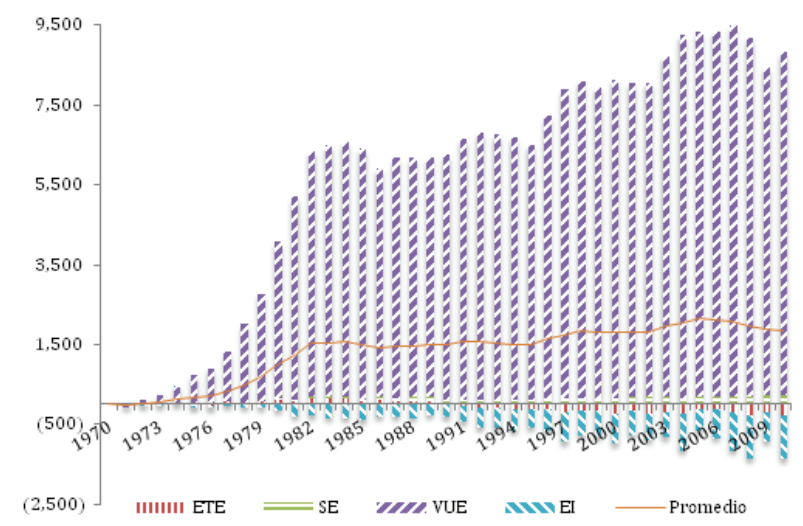

Figura 2. Distribución de efectos que explican los cambios en el consumo de energía total (PJ) 
Tabla 9. Evolución en la distribución de los efectos que explican el consumo de energía en los centros de consumo (PJ)

\begin{tabular}{lllllllllllll}
\hline \multicolumn{3}{c}{$\Delta$ EP } & \multicolumn{3}{c}{ ETE } \\
\hline Ex & 1980 & 1990 & 2000 & 2010 & 1980 & 1990 & 2000 & 2010 & 1980 & 1990 & 2000 & 2010 \\
CNE & $1,875.8$ & $2,822.6$ & $3,632.0$ & $3,428.5$ & 7.0 & -13.9 & -35.6 & -11.4 & -31.8 & -34.6 & -34.5 & -32.1 \\
R & 160.2 & 328.0 & 155.5 & 164.8 & -4.1 & -6.0 & -10.0 & -13.8 & 1.5 & -0.2 & -1.4 & -2.8 \\
T & 252.4 & 526.8 & 621.0 & 797.1 & 27.1 & 21.2 & -8.2 & 0.9 & 21.3 & 37.6 & 79.3 & 120.9 \\
A & 684.9 & 907.2 & 951.3 & $1,149.2$ & 16.4 & -20.7 & -103.2 & -164.8 & 3.8 & 0.5 & -1.3 & 0.1 \\
Ec & 85.8 & 97.1 & 108.2 & 132.4 & 4.4 & 1.0 & -8.3 & -8.4 & 4.9 & 17.8 & 15.5 & 12.8 \\
\hline Total & 890.5 & $1,269.7$ & $1,703.1$ & $1,782.0$ & 73.1 & 20.4 & -49.0 & -74.6 & 28.6 & 91.5 & 114.0 & 128.1 \\
\hline
\end{tabular}

Tabla 9. (continuación)

\begin{tabular}{lllllllll}
\hline \multicolumn{7}{c}{ VUE } & \multicolumn{3}{c}{ EI } \\
\hline Ex & 1980 & 1990 & 2000 & 2010 & 1980 & 1990 & 2000 & 2010 \\
CNE & $2,224.7$ & $3,382.2$ & $4,409.5$ & $4,123.6$ & -324.1 & -511.1 & -707.5 & -651.6 \\
$\mathrm{R}$ & 166.4 & 358.9 & 194.1 & 228.4 & -3.5 & -24.7 & -27.2 & -47.0 \\
$\mathrm{~T}$ & 155.6 & 358.6 & 453.9 & 488.0 & 48.4 & 109.4 & 96.0 & 187.3 \\
$\mathrm{~A}$ & 640.1 & 970.8 & $1,352.5$ & $2,058.5$ & 24.5 & -43.4 & -296.7 & -744.5 \\
Ec & 64.3 & 59.9 & 91.3 & 130.3 & 12.2 & 18.4 & 9.8 & -2.3 \\
\hline Total & 695.8 & $1,022.8$ & $1,479.6$ & $1,568.1$ & 93.0 & 135.0 & 158.5 & 160.3 \\
\hline
\end{tabular}

hasta 1993 y de ahí en adelante ha crecido, los sectores económicos crecieron rápidamente en la primera década y después con ligeras variaciones continuaron creciendo con menor intensidad (figuras $3 a, b$ y c).

De manera contraria los sectores exportación, CNE y transporte, el valor de SE son prácticamente nulos; al igual que los valores de ETE de 1970 a 1990, después toman valores negativos, excepto para el sector exportación donde el valor fue cero para todo el periodo; el efecto EI es negativo para estos tres sectores y entre la segunda mitad de la década de los 80 y la primera de los 90 aumentó su valor, se aprecia con claridad en el sector transporte; las trayectorias del efecto VUE son distintas para todos, para el sector exportación creció exponencialmente en la primera década del estudio, y en las siguientes se ha mantenido lineal con variaciones de 500 PJ, para el sector CNE creció rápidamente en las primeras dos décadas, en las siguientes dos décadas disminuyó su valor abruptamente, el sector transporte también creció con rapidez en la primera década, las siguientes dos décadas se mantuvo prácticamente constante y en la última creció 500 PJ (figuras 3d, e y f).

\section{Conclusiones}

La producción y el consumo de energía en México están concentrados en los hidrocarburos y sus subproductos, lo cual explica la cantidad de recursos energéticos que tiene y el grado del rendimiento energético.
Para las fuentes de energía primarias, como el petróleo crudo y el gas natural, la relación es de 195 y 12 unidades de energía, respectivamente. Las fuentes secundarias, como los derivados del petróleo crudo, tienen valores entre uno y dos unidades de energía, menor a uno para el caso de la electricidad, recordando que esta es una fuente de energía secundaria y terciaria.

De la información obtenida en el cálculo de los $\overline{E P}_{\text {directa }}$, se concluye desde el punto de vista termodinámico, que la entropía del sistema energético mexicano va en aumento, debido la acumulación de irreversibilidades asociadas a los procesos de conversión de energía, teniendo como causa de ellas la producción de subproductos de hidrocarburos y su consumo en los sectores económicos y el transporte.

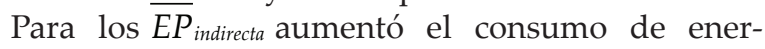
gía para mover energía, dado que la infraestructura del sistema energético no asimiló el crecimiento exponencial en la producción de hidrocarburos de la década de los 70, alcanzando en 1979 el punto de inflexión donde se convierte en el principal consumo, lo que marca un cambio estructural energético. Tiende a los hidrocarburos como los principales energéticos disipados y los sectores de exportación, económicos y residencial como los más sensibles a este tipo de consumo.

Al estimar los cambios en el consumo de energía total, $\overline{E P}_{\text {total }}$, se observa que el sector exportación tiene una relevancia de $46 \%$ en la estructura de consumo de energía. Desde el punto de vista de la sustentabilidad, esa 

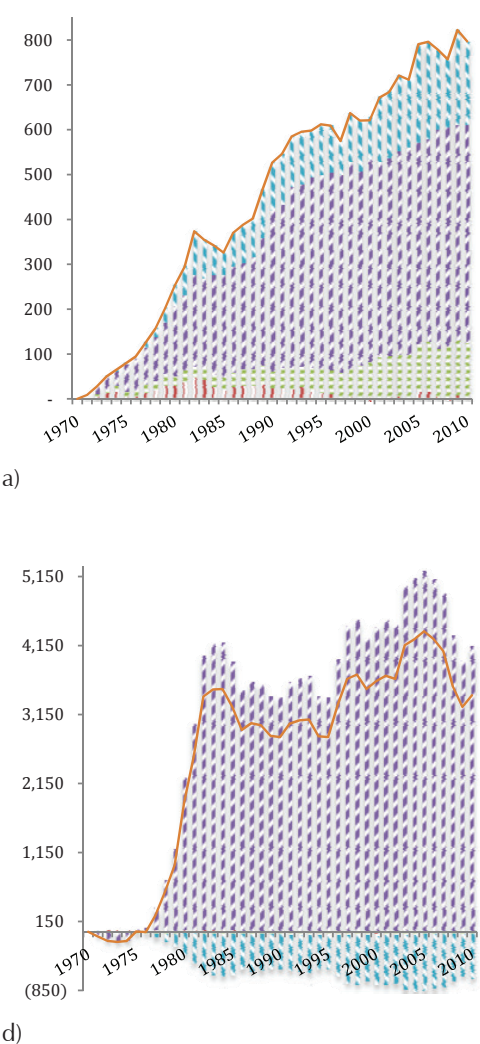

IUU口 ETE

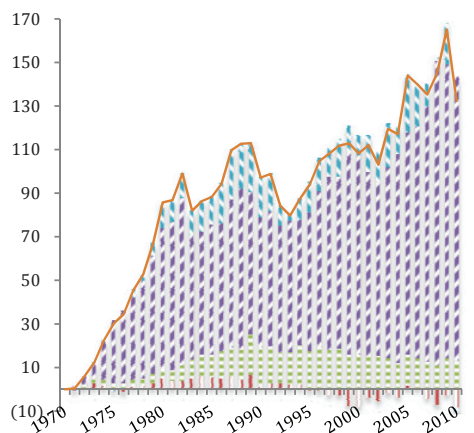

b)

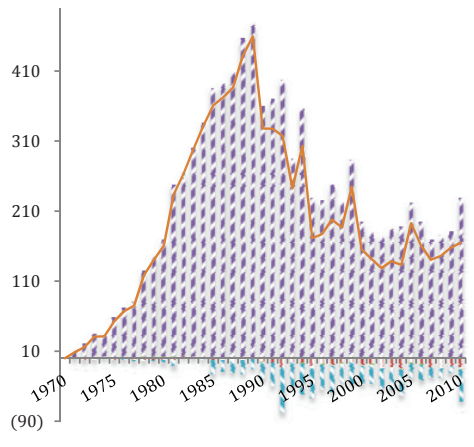

e)

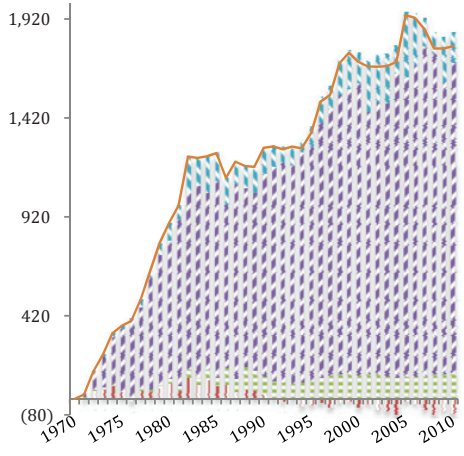

C)

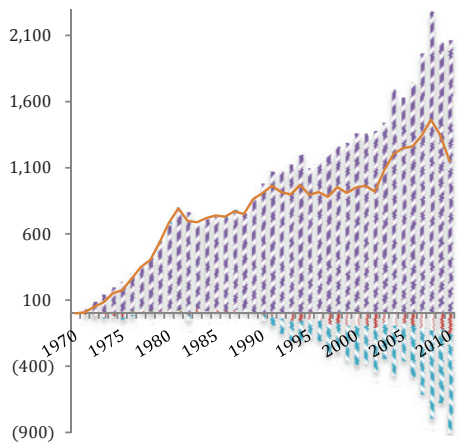

f)

Figura 3. Distribución de efectos que explican el consumo de energía (PJ), a) residencial, b) agropecuario, c) sectores económicos, d) exportaciones de energía, e) sectores de consumo no energético, f) transporte

energía no asimilada tiene efectos negativos en los demás sectores, si plantean un crecimiento en su producción estos no tendrán energía para sustentarlo, las opciones que tiene son diversificar sus fuentes e importar energía, lo que conlleva al aumento de sus costos de producción. Por lo tanto, se concluye que la salida de esa cantidad de energía resta capacidades de aumentar el efecto actividad en los sectores más dependientes de los hidrocarburos.

El ACE demuestra que los principales cambios en el consumo de energía son para los sectores exportación, económicos, residencial y transporte. El efecto VUE es el principal en todos los sectores, es decir, la actividad de cada uno de ellos, concluyendo que el consumo de energía es directamente proporcional a este efecto.

Además revela que los cambios por los efectos de eficiencia de conversión y diversificación energética han tenido poco peso. La importancia de esto radica en que la forma y la intensidad de hacer mercado explica el nivel de consumo y simultáneamente invita a reflexionar acerca de la estructura de la oferta energética para cubrir las necesidades de los sectores, sobre todo en aquellos en los que el efecto SE ha estado presente directamente.

Se considera que las diferentes formas de energía que utiliza el sistema, los coeficientes de transformación de energía y el estado de la infraestructura de transmisión-distribución y almacenamiento de energía, son los elementos clave para la gestión energética nacional que contribuya al desarrollo sustentable.

La aportación de la energía para alcanzar criterios de desarrollo sustentable radica en cubrir los requerimientos de energía del propio sistema y de todos los sectores consumidores, de manera que disminuyan los coeficientes de transformación de energía, ya sea por factor tecnológico o por reducir los pasos de conversión y por mejoras en la infraestructura de transmisión-distribución y almacenamiento de energía, disminuyendo la distancia recorrida, la calidad de sus materiales y dispositivos de control.

Los criterios de sustentabilidad como la temporalidad de los recursos y el deterioro ambiental, pone en 
cuestión la tendencia a centralizar los recursos energéticos en los hidrocarburos, ya que de no mantener los niveles de extracción, los valores del rendimiento energético caerán, por lo que romper con esa tendencia es clave para la sustentabilidad del sistema energético mexicano.

Aumentar el peso proporcional de las demás fuentes en la matriz energética tiene efectos positivos simultáneos, baja la carga de los hidrocarburos en el sistema, lo que significa descarbonizarlo, es decir, el menor consumo de hidrocarburos es una defensa contra el deterioro ambiental (vía menos moléculas de carbono), al mismo tiempo aumenta la eficiencia de conversión energética al disminuir los pasos para transformar la energía disponible en útil.

\section{Referencias}

Alcántara V. y Roca J. Energy and CO2 emissions in Spain. Methology of analysis and some results for 1980-1990. Energy Economics, volumen 17 (número 3), 1995: 221-230.

Flores-Velázquez R., Muñoz R.L.C., Villalba-Valle D. Inventario de emeisiones en 2005 de gases de efecto invernadero por el sector energético mexicano. Ingeniería, Investigación y Tecnología, volumen X (número 1), 2010: 35-43.

Johanson T., y Goldemberg J. Energy for Sustainable Development, United Nations Development Programme (UNDP), Nueva York, 2002.

Miller R. y Blair P. Input-output analysis; foundations and extensions, 2a ed., volumen IX, Nueva York, USA, Cambridge University Press, 2009.
Proops J.R.J. Energy Intensities, Input - Output Analisys and Economic Development; en Ciaschini, M. Input - Output Analisys. Current Development, Chapman and Hall, 1a ed., pp 201-216.

Pruitichaiwiboon P., Lee C.K., Baek C.Y., Lee K.M. Development and application of an energy input-output table for an energy demand and supply activities analysis. Enviromental Engineering Research, volumen 16 (número 1), 2011: 19-27.

Slesser M. Net energy as an energy planning tool. Energy Policy, junio de 1987.

SENER. (n.d.). Energia. Retrieved 10 de enero de 2013, Sistema de Información Energética (SIE) [en línea]. Disponible en: http://sie.energia.gob.mx.

Sheinbaum C.P., Rodríguez V.P., Robles G.M. Política Méxicana e Indicadores de Sustentabilidad. Problemas del Desarrollo, volumen 40 (número 158), 2009: 113-135.

UNDESA. Our Common Future, 1987. Retrieved 11 de octubre de 2013 from Unated Nations [en línea]. Disponible en: http:// www.un-documents.net/our-common-future.pdf

\section{Este artículo se cita:}

\section{Citación estilo Chicago}

Livas-García, Adrián. Analisis de insumo-producto de energía y observaciones sobre el desarrollo sustentable, caso mexicano 1970-2010. Ingeniería Investigación y Tecnología, XVI, 02 (2015): 239-251.

\section{Citación estilo ISO 690}

Livas-García A. Analisis de insumo-producto de energía y observaciones sobre el desarrollo sustentable, caso mexicano 1970-2010. Ingeniería Investigación y Tecnología, volumen XVI (número 2), abril-junio 2015: 239-251.

\section{Semblanza del autor}

Adrián Livas-García. Es ingeniero en energía por la Universidad Autónoma Metropolitana-I, tiene estudios de maestría en ingeniería en energía por la Universidad Nacional Autónoma de México, actualmente es candidato a doctor en ingeniería en energía por la misma institución. 